\title{
Formulación, implementación y evaluación de políticas públicas desde los enfoques, fines y funciones del Estado*
}

Formulation, implementation and evaluation of public policies from the approaches,

purposes and functions of the State

Jahir Alexander Gutiérrez Ossa ${ }^{1 \mathbf{x}}$

Rubén Darío Restrepo Avendaño $0^{2 \otimes}$

John Stivens Zapata Hoyos ${ }^{3 凶}$

Fecha correspondencia:

Recibido: 16 de Agosto de 2017.

Revisión: 29 de septiembre de 2017.

Aceptado: 15 de noviembre de 2017.

Forma de citar:

Gutiérrez, J.A., Restrepo, R.D.,

Zapata, J.S. Formulación,

implementación y evaluación

de políticas públicas desde los

enfoques, fines y funciones del

Estado. Revista CES Derecho, (8), 2,

333-351.

Open access

Términos de uso

Licencia creative commons

Etica de publicaciones

Revisión por pares

Gestión por Open Journal System

DOl: http://dx.doi.org/10.21615/

cesder.8.2.7

ISSN: 2145-7719

Sobre el artículo:

* Esta ponencia es producto del proyecto de investigación:

Perspectivas del conflicto, la política,

los recursos naturales y la vecindad

Intermunicipal en la Subregión

del Oriente Antioqueño desde las

Trampas del Subdesarrollo. Estudios

de casos institucionales: 1. Formas

de Asociatividad Territorial en el

Oriente Antioqueño y; 2. Construcción

\section{Resumen}

El artículo plantea la hoja de ruta en materia de formulación, implementación y evaluación de políticas públicas. Los fines y las funciones del Estado, representan las fuentes y orientaciones respectivamente, sobre las que deben basarse las políticas públicas. Se recurre a los métodos descriptivo y exploratorio para dar cuenta de la perspectiva sistemática de las políticas públicas, a su vez, que la revisión metodológica partirá de los enfoques de planes, política económica, programas y/o proyectos y secuencial para establecer el grado de naturalización de las mismas. El primer producto a desarrollar, está encaminado a exponer los elementos que requieren las políticas públicas para materializarse; como segundo, las guías que deben tener las políticas públicas para la interacción entre los sistemas de ordenamiento, y por último, la revisión sobre los enfoques de las políticas públicas, frente a los fines y funciones del Estado.

Palabras claves: Enfoques de derechos, politica pública, evaluación, límites y restricciones, tipos de política.

\section{Abstract}

The article raises the roadmap as for formulation, implementation and evaluation of public policies. The ends and the functions of the State, they represent the sources and orientations respectively, on that the public policies must be based. One resorts to the methods descriptively and exploratory to realize of the systematic perspective of the public policies, in turn, that the methodological review will depart from the approaches of plans, economic politics, programs and / or projects and sequentially to establish the degree of naturalization of the same ones. The first product to developing, is directed to expose the elements that need the public policies to materialize; as I come second, the guides who must have the public policies for the interaction between the systems of classification, and finally, the review on the approaches of the public policies, opposite to the ends and functions of the State.

Key words: Approaches of rights, public policy, evaluation, limits and restrictions, types of policy. 
de los programas de desarrollo

con enfoque territorial (PDET)

en los municipios priorizados

del posconflicto. Código

INV022017004. Realizado por

el Grupo de Investigación en

Gestión Empresarial de la

Universidad CES y el Grupo

de Investigación FACEA, de la

Universidad Católica de Oriente.

Fecha de inicio: 26/04/2017

y Fecha de terminación:

6/10/2018. Linea: Teoria y

pràctica de la implementación

de políticas públicas.

Sobre los autores:

1. Comisionado de

Ordenamiento Territorial para

el Departamento de Antioquia

(2015-2017). Economista de

la Universidad de Medellín,

Colombia, Estudiante

Doctorado Desarrollo

Sostenible, Universidad de

Manizales, Caldas. (PHD)

en Administración Pública,

Atlantic International

University (AIU), Honolulú,

USA, Magíster en Desarrollo,

Universidad Pontificia

Bolivariana, Medellín,

Colombia. Economista

Industrial de la Universidad

de Medellín, Colombia.

Coordinador del Grupo de

Investigación en Gestión

Empresarial de la Universidad

CES. Facultad de Ciencias

Administrativas y Económicas.

Tel.: 4440555-1411. Dirección:

Calle 10 A \# 22-04.

2. Médico Cirujano,

Universidad de Antioquia,

Magíster en Salud pública,

Universidad CES Medellín.

Medellín - Colombia.

3. Estudiante Administración

de empresa, Sexto semestre

- Universidad CES. Monitor

línea de investigación y

Finanzas (Análisis financiero)

\section{Introducción}

Es del resorte estatal el auspicio, fundamento y proliferación de las políticas públicas, contrario a la postura anti estatal sobre la que se pretende que estas sean desplegadas, es decir, que surjan como instrumentos causales de decisiones sobre intervención gubernamental, sin haber sido consideradas exactamente en procesos de planeación. Por ende, el artículo está enfocado a exponer la orientación que deben tener las políticas públicas, asimismo que el trasegar de estas con respecto no sólo a sus enfoques, sino a la manera como deben entenderse desde la perspectiva de los fines y funciones del Estado. No obstante, las políticas públicas tienden a ser cíclicas, por lo tanto, no cabe asistencialismo solo como acción de correción e intervención específica que carezca de perdurabilidad, siendo estos dos patrones que descuidados, terminarían por reducir el carácter cíclico de la política pública, y por ende, el margen de ajuste, corrección, diseño o promoción de las mismas.

La orientación dada en cuanto a la formulación, implementación y evaluación de la política pública, implica no sólo la concreción del tema a facultar como tal o las áreas ha abrigar en ella, sino la trazabilidad compleja, sistémica y léxico pragmática que esta contiene. No todo puede ser política pública en fines estatales, reconociendo así que se deben realizar planeaciones en otras temáticas análogas al fin del Estado. Empero, para que la política pública responda a los criterios básicos de que sea general, no militante y convergente, se debe tener claro los alcances, propósitos y resultados que se espera que arrojen éstas, a las que les cabe una guía que oriente su adecuada formulación a pesar de los intereses politicos propios que puedan existir de por medio en su configuración. El origen político, no es óbice para exponer la base de su formulación, menos el marco institucional que las acoge, y mucho menos, la evaluación que hace posible su exposición o monitoreo.

En cuanto a los enfoques planteados en las políticas públicas, diseminados en planes, política económica, programas y/o proyectos y a escala secuencial, es de aclarar que la política pública presenta diversos matices, unas de naturaleza constitucional, otras de orden técnico, algunas extendidas en temas de interés público internacional y un tanto, familiarizadas con la ejecución y/o la participación. No obstante, se considera por lo general, que las políticas públicas tienden a reivindicar temas en cuestión de asuntos públicos sobre los cuales sus actores presentan competencias en ellos, cuando las políticas públicas tratan en esencia sobre mejorar estos temas más que la simple reinvindicación. Prevalece cierto orden entre ellas, pero lo que es cierto, es que las políticas también requieren ser planeadas, por ende, se asume la prevalencia de este último término por encima de la discreción política.

El papel que juega en particular los fines y las funciones del Estado, da cuenta del alcance, impactos y resultados a la par que la maniobrabilidad o versatilidad de las políticas públicas, precepto en el que cabe el grado de relación contractual o extracontractual del Estado frente a estas. Es importante reconocer, que las políticas públicas atienden al derecho, la legislación, a la población y el territorio, en tanto mejoran la asignación de recursos, la distribución de la riqueza, la estabilidad económica y el fomento al desarrollo. Es claro que para el Estado es contraproducente exponer sus fines al escrutinio de las políticas públicas, no obstante la intención de hacerlo, implica que éste es capaz de anteponerse, para que las cosas mejoren.

Lo introducido en el marco de la temática central lleva a plantear los siguientes capítulos: 1. Elementos para la orientación que deben tener las políticas públicas a la hora de formularse, implementarse y evaluarse; 2 . Guías de los planes, la política 
Facultad de Ciencias

administrativas y económicas

- Universidad CES. Integrante

Grupo de investigación

Gestión empresarial, línea

de Estrategia, Dirección y

reputación corporativa. económica, los programas y proyectos, a la par que al enfoque secuencial para la estructuración de políticas públicas; y 3. La evaluación de los enfoques de la política pública frente a los fines y funciones del Estado, frente al ciclo de las políticas; además, del vínculo contractual y extracontractual que generan. Las políticas públicas, son instrumento valioso, que de formularse, implementarse y evaluarse bajo una justa medida, harían posible exponer los alcances, límites y restricciones del Estado.

\section{Elementos para la orientación de la formulación, implementación y evaluación de las políticas públicas}

El postular una guía que oriente la formulación, implementación y evaluación de políticas públicas, podría en principio, no generar mayor debate. No obstante, el plantear la idea de que estas deben ser consistentes con el momento de la planeación, la población, la ordenación del suelo y las condiciones territoriales, implica una revisión distinta a lo pretendido por lo general. Por ende, las políticas públicas deben emerger de la consistencia entre la presencia de sistemas de ordenamiento de suelos y del territorio, de planeación y población, dado que la política se desarrolla en cada uno de dichos ámbitos sistemáticos.

Cuando se pierde el sentido político de las políticas públicas y éstas se asimilan a cualquier instrumento de intervención gubernamental, orientada ha resolver problemas públicos, haciendo abstracción del proceso de formulación de los actores que deben participar en él para incrementar la legitimidad de la decisión. Jolly \& Cuervo (2007, p. 15-16).

Al igual, que el resto de tipologías similares, las políticas públicas no son temáticas en particular a las que ha de apuntarse, surgen por cuenta del grado de evolución que han tenido los escenarios básicos en donde estas tienen acceso. Por ello, es que en ocasiones se confunden con otras políticas, en tanto, mecanismos reivindicatorios, de resarcimiento, mejoramiento o para esquilmar al Estado. Lo que es cierto, es que las políticas públicas deben tener un origen cohonestado con la realidad del espacio - lugar al que se pretenden coligar, dado que se considera que las políticas públicas, superan a veces la realidad.

Pocos economistas consideran la política económica como una parte del conjunto conformado por la "ciencia de las políticas públicas" (Policy Science) o, de una manera más restrictiva, como un posible campo de aquella. (Jolly, 2007, p. 131).

La disposición de planes, políticas, programas y/o proyectos y escalas secuenciales, implica la prevalencia de iniciativas diversas para engrosar las políticas públicas. No obstante, cada una de ellas debe igualmente abocarse a que sean generadas en el marco de la concomitancia y existencia de sistemas de ordenamiento, población, suelos y territorio, enquistados en los marcos jurídicos, para que las iniciativas de políticas públicas tengan trascendencia estructural, y que con cargo a ello, sean revisados a través de los sistemas, en tanto, estos terminan por orientar la tipología de las políticas públicas.

¿Cómo ingresa un problema público en la agenda? Como se dijo en el enfoque sistémico, son los políticos los que hacen la primera selección de los problemas que deben ingresar en la agenda pública. (Cuervo, 2007, p.157).

La orientación en cuanto a política pública, subyace en la medida que prevalezcan sistemas de planeación, de población de ordenamiento y de suelos, en particular, que prioricen de manera articulada la concreción, definición o postulación de una 
política pública en sentido estricto. De lo contrario, se desdice de las cualidades que debe tener una política pública, en tanto, que sea general, no militante, que sea convergente y que no desdiga o reproduzca una política existente, patrones que por demás, influyen en la afluencia de estas por doquier, periplo particulariza la complejidad común de la política pública dado los factores que la integran.

\section{Clases de agenda}

La agenda sistémica es el conjunto de problemas que aún no han sido seleccionados por parte del sistema político, es decir, todos los problemas de una sociedad. La agenda institucional es cuando ya el sistema político ha hecho una primera selección, pero aún no ha sido transmitida al gobierno. La agenda gubernamental es la agenda que elabora el gobierno con los insumos del sistema político y para el que asigna los recursos orientados a su tratamiento en la lógica de políticas públicas. Pero dentro esas agendas también aparece la agenda política, que es la agenda que conserva el sistema político. De otra parte, tenemos la agenda de largo plazo o agenda estratégica y la agenda contingente, cuya combinación es la que permite a los gobiernos modular las necesidades de resolver problemas. (Cuervo, 2007, p.163).

La formulación aparece por cuenta de la propia integración o movilidad de los planes o programas etc., es decir, que responden a un comportamiento estructural de los planes administrativos o territoriales, que de manera concomitante y recíproca no enaltece una gestión en particular sino el derrotero estamental. En esencia, si los planes no son convergentes, las políticas públicas tampoco, por eso, es algo extraño que permanezcan políticas públicas en planes no concurrentes, prueba de la complejidad aleatoria de las políticas por momentos, que en ocasiones no parece que tuvieran principio o finalidad o largo aliento, pero que para su conveniencia deben tener ciclo de cierre o renovación continua.

La emergencia de la noción de red (network) en el análisis de las políticas públicas corresponde a la insatisfacción creciente en relación con los enfoques tradicionales centrados en el examen de los elementos formales de las estructuras y arreglos políticos. (Roth, 2007, p. 43).

El concurrente llamado a denominar todo lo social, en especial como política pública, ha desvirtuado la capacidad integradora, interdisciplinaria y polifacética de esta. La política pública puede responder a intereses de intervención, interinstitucionales o sectoriales, facultativos o procedimentales para allanar espacios no contemplados o fuera de foco. Por ende, la formulación contiene no sólo el rastreo de la tipología de política pública, sino el grado de convergencia que logra tener frente a los sistemas creados en el territorio, explica la naturaleza simbiótica de los sistemas y la propia política pública, que por contadas razones implica que esta debe provenir de un juicio sistémico de lo que sucede para poder generarlas.

Las redes de actores establecen un puente entre las jerarquías de administrativas y la lógica del mercado. El Estado ya no sabe todo y no es capaz de saberlo todo, y menos aún de imponerse en la sociedad mediante un proceso de implementación vertical de tipo comando - control. Se produce un proceso de reconocimiento de necesidad mutua de los actores. Roth (2007, p. 44).

El ejercicio de la implementación de las políticas públicas aflora en la medida en que se tenga clara la magnitud de estas. En tanto, la versión de dicha apuesta a escala global o internacional, en los organismos multilaterales o estamentales a la par que 
con organismos de dirección, gestión o intervención como la Organización de las Naciones Unidas, el Banco Mundial o el Fondo Monetario Internacional, entre otros. La implementación implica, el grado de conocimiento, generalidad y simultaneidad temática de las políticas públicas. Las cifras, datos, lineamientos y orientaciones, fundamentan la implementación, en razón del papel que cumplen dichos elementos para dar paso a los normales ajustes o revisiones que requieren.

La construcción de un enfoque que retomaría la noción de hábitos, en su intento de articular los condicionamientos estructurales que pesan sobre los agentes y de tomar en consideración su capacidad creativa, limitada por su experiencia social, asociada con el concepto de cambio, como espacio de posicionamiento de los agentes en la estructura social de la política considerada, podría entonces abrir nuevas pistas analíticas. Roth (2007, p.53).

El manejo de las estrategias Bottom - Up, Co-Working o Top-Down, expresan de manera directa la forma como ha de incubarse la política pública luego de ser formulada. El Bottom - up, pone a la población en el centro de la política pública como hacedora, ejecutora pero igualmente responsable de la misma, sobre lo cual debe dar rendición de cuentas. A su paso, el co-working, implica la capacidad de concurrencia, redes o subsidiariedad de quienes las formulan, en tanto, en el Top - Down, las encabezan quienes tienen carácter direccional. En ningún caso, la estrategias son inamovibles, contrariov a ello, son cíclicas y por ello, a pesar de la manera como se implementen inicialmente, estas pueden cambiar conforme a prioridades.

Tabla 1. Instrumentos complementarios del estado para la operatividad de las políticas públicas

\begin{tabular}{ll}
\hline \multicolumn{1}{c}{ Instrumento } & \multicolumn{1}{c}{ Características } \\
\hline Prescriptivos & Obligación. Interdicción. Autorización \\
\hline Incentivos & No obligatorio. Induce conducta. Recompensas \\
\hline De coordinación & Planificación. Estudios de impacto \\
\hline De organización y de & Definen el marco de acción y el proceso en el cual se \\
procedimiento & desarrollará la política pública \\
\hline Materiales & Estado provee directamente bienes/servicios \\
\hline De delegación a sociaos & Entidades del Estado colaboran con privadas y Tercer Sector \\
\hline Fuente: Elaborado con base a Roth (2004). & en llegar a objetivos de políticas \\
\hline
\end{tabular}

Al entrar la evaluación de la política pública en la medida de los campos, propósitos o temáticas a destacar, es importante que se entienda que ésta no sólo pasa por el ajuste o conciliación entre lo accionado, ejecutado y obtenido. La política pública, debe contar con un alcance interinstitucional e igualmente de alcance global, precisamente, para que esta misma sea garante de una revisión coherente a lo matizado en el marco de características de las políticas públicas, en tanto, los impactos y resultados obtenidos en razón de ellas. Es decir que, la propia política pública dispone de elementos para auto ajustarse. No obstante, está depende de la intención que se tiene para con la puesta en marcha de la política de manera directa o indirecta, y los espacios que se pretenden corroborar o fortalecer a través de ellas.

Tabla 2. TIPOLOGÍA DE LOWI' Los Cuatro tipos de Lowi

\begin{tabular}{ccc}
\hline Cuatro tipos LOWI & Comportamiento & Entorno \\
\hline Indirecta o lejana & Política Distributiva (1) & Polít. Constitutiva (2) \\
Directa & Polít. Reglamentaria (3) & Pol. Redistributiva $(4)^{5}$ \\
\hline
\end{tabular}

Fuente: Elaborado con base a Roth (2004) 
En la evaluación, deben enquistarse índices, indicadores, categorías o factores a la par que variables a través de los cuales, la política pública pueda dar cuenta de la convergencia de sistemas que le favoreció, a su vez, que sobre el grueso de propósitos para la cual fue creada. No obstante, la evaluación debe obedecer a criterios complejos en la medida que está expuesta a correcciones, mitigaciones o riesgos, a su vez, que a un contenido sistémico, en la medida que muestra sus logros, y de los sistemas que la exponen. La evaluación, subraya la planeación, política, programa o proyecto y secuencia de las políticas públicas, en la medida que permite de manera esporádica, revisar los componentes de las políticas públicas.

Tabla 3. Componentes de evaluación de política pública

\begin{tabular}{ccccc}
\hline Índices & Indicadores & Categorías & Factores & Variables \\
\hline Globales & Largo plazo & Políticas globales & cualitativo & (Impactos y Resultados) \\
\hline Mundiales & Mediano plazo & Programas mundiales & $\begin{array}{l}\text { Cualitativo/ } \\
\text { cuantitativo }\end{array}$ & $\begin{array}{c}\text { (Externalidades y } \\
\text { Supuestos) }\end{array}$ \\
\hline Internacionales & Corto plazo & Planes internacionales & cuantitativo & (Costo /Beneficio) \\
\hline Fuente: Elaboración propia. & & & &
\end{tabular}

Lo atinente a la planeación y la población, cuya naturaleza es mucho más cualitativa y estética que la contenida en el primer grupo de sistemas. Es importante considerar el componente cualitativo que distingue a este grupo de sistemas de planeación y población, desde las que efectivamente se destacan los impactos promovidos en la política pública de la mano con ellos. En este escenario, aparecen los índices, cuya idea arrastra la posibilidad de evidenciar con claridad qué tanto las políticas públicas permean la planeación y la población, en las que se enmarca la idea que los índices perfilan los sistemas. Lo que es claro, es la sinergia sistémica entre los enfoques de política pública que se plantean cómo enfoques.

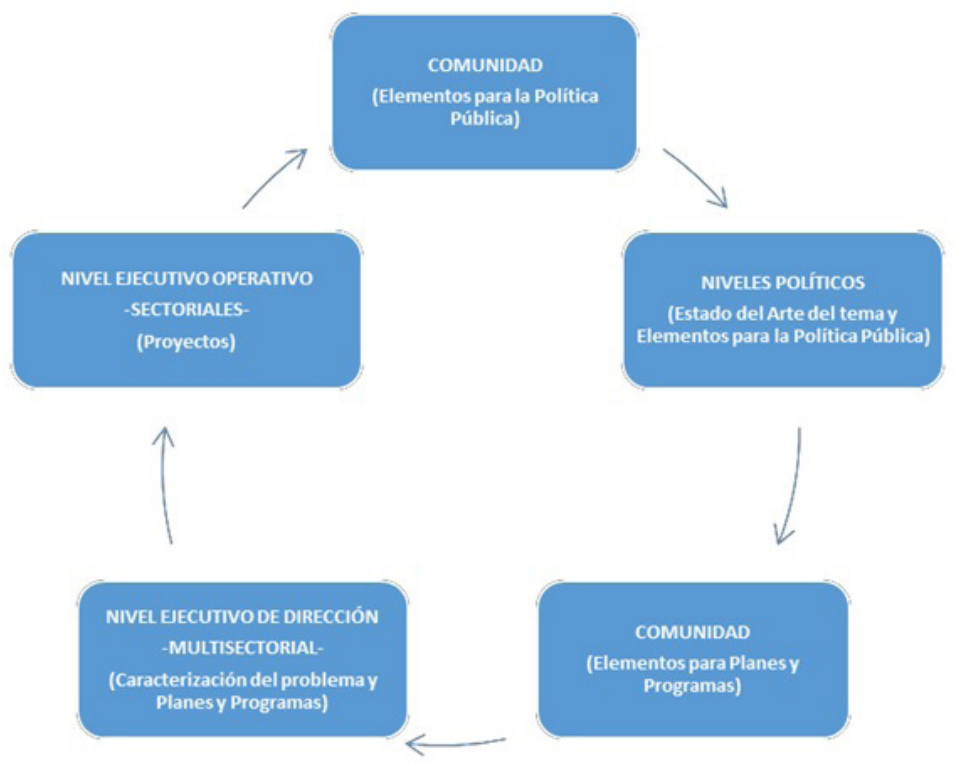

Esquema 1. Círculo de la formación de elementos y de políticas públicas, planes, programas y proyectos

Fuente: Elaboración propia

En cuanto a la respuesta que deben tener los sistemas expuestos, en primer lugar, el de ordenamiento y de suelos, dan cuenta de la provisión del recurso espacial - geográfico con el cual cuenta la administración para favorecer ciertas políticas públicas. 
Es decir que, es el lugar de encuentro es con la misma naturaleza territorial y las posibilidades de acceso, incubación y progreso de las políticas públicas. Como segundo lugar, el sistema de ordenamiento y suelos, desde la condición física del territorio rinden cuentas por medio de indicadores, y en razón a las políticas públicas con la realidad que deben enfrentar estas, dado que deben cumplir los mismos principios que los procesos de planeación territorial.

\section{Guía de los Planes, política económica, programas, proyectos y análisis secuencial para formular, implementar y evaluar políticas públicas}

La acción estatal se distingue por la vía de los planes en sus diversas connotaciones, la política económica enmarca en la dirección productiva, los programas orientados a problemas estructurales a la vez que de proyectos dirigidos a las ejecuciones, y en su momento en el análisis secuencial para dar cuenta del liderazgo de los proyectos en cabeza de los actores o poblaciones. Básicamente, no toda intervención es política pública, pero, toda acción estatal busca mejorar la situación de distintos agentes a través de las fuentes en las que se encumbran las políticas públicas, como las referidas en este documento. En este ámbito, la revisión de cada una de ellas, hace posible aventurarlas como planes, programas o proyectos.

La aplicación de supuestos económicos en el estudio de las políticas públicas permite abstraer las variables más relevantes para construir una serie de modelos que generen predicciones sobre las elecciones que realizan los individuos y las sociedades en los mercados. Sour (2008:3).

En términos generales, los planes enfocados hacia el desarrollo representan el primer muestrario de acción de políticas públicas. Su carácter constitucional, que abarca obligatoriedad en su planteamiento, revisión y puesta en marcha para beneficio de la población, expone el orden institucional y la dirección de los estamentos que lo llevan a cabo. En su estructura diagnostica o estratégica al igual que en su fundamento financiero o presupuestal, se expone claramente las intenciones que se tiene en diversos frentes y la manera de realizarlos, por ende, cumple por completo los patrones característicos de la política pública, de generalidad, no militancia, vinculante y que conversa con otras políticas.

Tabla 4. Composición de los planes de desarrollo como política pública.

\begin{tabular}{ll}
\hline Estructura general o estratégica & Financiera o presupuestal \\
\hline Diagnóstico & Marco Fiscal de Mediano Plazo \\
Problemas & Margo de Gasto de Mediano Plazo \\
Estrategias & Plan Anualizado de Caja \\
Líneas estratégicas & Plan Mensualizado de Caja \\
Programas & Plan Operativo Anual de Inversiones \\
Subprogramas & Plan de acción \\
Proyectos & Plan de ejecución \\
\hline Fuente: Elaboración propia &
\end{tabular}

Al identificar la composición de los planes de desarrollo, se entiende la estructura que debe contener una política pública proveniente del orden estamental. No obstante, como el resto de políticas públicas, debe ser por cuenta de la estructuración de sistemas de planeación, ordenamiento, suelos y población, entre otros que se requieran 
categorizar, para que de ellos emerjan las políticas públicas. Es claro, apreciar que estas tienen un comportamiento cíclico o que dependen de circunstancias especiales para que emerjan como una posibilidad, en la que medida que deriven desde un orden complejo o sistemático. Los planes, son ejercicios territoriales, que deben superar el carácter misional al que están siendo abocados.

Tabla 5. Definición de políticas públicas bajo la concepción de sistemas

\begin{tabular}{|c|c|c|c|c|}
\hline $\begin{array}{l}\text { Sistema de } \\
\text { planeación }\end{array}$ & $\begin{array}{c}\text { Sistema de } \\
\text { ordenamiento }\end{array}$ & Sistema de suelos & Sistema poblacional & $\begin{array}{l}\text { Políticas } \\
\text { Públicas }\end{array}$ \\
\hline Administrativa & $\begin{array}{l}\text { Político } \\
\text { administrativa }\end{array}$ & $\begin{array}{l}\text { Composición } \\
\text { territorial }\end{array}$ & Pirámide poblacional & Poblacionales \\
\hline Estratégiga & Vecindad & $\begin{array}{l}\text { Características del } \\
\text { territorio }\end{array}$ & Demografía & Dimensionales \\
\hline Legislativa & $\begin{array}{l}\text { Base, media y } \\
\text { alta }\end{array}$ & $\begin{array}{l}\text { Grado de } \\
\text { convergencia }\end{array}$ & Oferta poblacional & $\begin{array}{l}\text { Impactos y } \\
\text { resultados }\end{array}$ \\
\hline Perspectiva & Escenarios & $\begin{array}{l}\text { Límites y } \\
\text { restricciones }\end{array}$ & $\begin{array}{l}\text { Demandas de la } \\
\text { población }\end{array}$ & Futuros posibles \\
\hline Sistémica & $\begin{array}{l}\text { Auto } \\
\text { organización }\end{array}$ & Capacidad de carga & $\begin{array}{l}\text { Costos sociales de la } \\
\text { transacción }\end{array}$ & Concurrencia \\
\hline
\end{tabular}

En cuanto a la política, particularmente económica y enfocada al desarrollo, es evidente que las políticas públicas tienen un carácter sumamente sectorial o estratégico que obedece a un trabajo mancomunado con grupos de interés sobre los cuales la atención de los propósitos o resultados supera cualquier discrecionalidad o planteamiento puntual. En este ámbito, campos como la economía, la educación y la salud entre otros, deben abocarse a la materialización de propuestas de política pública, más que de cometidos legislativos, por cuanto, son propias del sector que las pretende llevar a cabo. La confluencia de factores políticos, no implican la presencia del Estado, por cuanto, los actores también son políticos.

Tanto la política como las políticas públicas tienen que ver con el poder social. Pero mientras la política es un concepto amplio, relativo al poder en general, las políticas públicas corresponden a soluciones específicas de cómo manejar los asuntos públicos. El idioma inglés recoge con claridad esta distinción entre politics y policies. Lahera (2004:7).

La política, hace referencia a la capacidad que tiene el aparato gubernamental, institucional y legal para favorecer la co-creación de políticas públicas, por ende, el matiz económico se convierte en elemento crucial para comprender la magnitud de las políticas que se alistan como material para la fusión con políticas públicas. Partiendo de la idea que, la política es el arte de hacer del bien a otros, se considera que independiente a su materialización como política cultural, económica, social entre otras, dentro de estas revisiones la más importante, es la que se hace a las demás desde la perspectiva económica. Por ello, es que debe tenerse en cuenta que la política no significa, léase al Estado, sino que está por encima de la política pública.

Tabla 6. Políticas y políticas públicas.

\begin{tabular}{ll}
\hline \multicolumn{1}{c}{ Políticas } & \multicolumn{1}{c}{ Políticas públicas } \\
\hline Económicas & Justo - precio de las políticas públicas, en cuanto a relación costo -beneficio. \\
Estratégicas & Abrir oportunidades en diferentes frentes. \\
Institucionales & Concurrencia y subsidiariedad. \\
Sociales & Equidad e igualdad. \\
Institucionales & Concurrencia y subsidiariedad. \\
Sociales & Equidad e igualdad.
\end{tabular}


La alianza entre la planeación y la política, permite establecer elementos de trabajo frente a los programas que se articulan a través de entidades, organismos o instituciones multilaterales o mundiales. Tradicionalmente, se trata de temáticas de interés general sobre el que se atienden de manera directa por la vía de las políticas públicas la intención concreta de dichos organismos y países receptores. En este frente, se infiere que los programas tienen un corte más transversal e internacional en tanto la ascendencia de los temas, por cuanto, terminan abrigados por diversos grupos de interés para sacarlos avante, por ello, la política pública debe ser transferible.

El contexto y las características de un programa son elementos importantes a considerar. Cuando la planeación de la evaluación se encuentra en la etapa de la selección de metodología, el análisis contextual y del objeto del programa debe haber sido ya agotado pues constituyen variables importantes para definir qué herramienta metodológica es más pertinente. Gris (2016:15).

El fundamento de los programas está dirigido a reconocer diversos actores, fuentes de apoyo y recursos a su vez que diferentes resultados. Se trata de una matriz de interesados de distinta índole que convergen para sacar al frente una temática. Tal vez, es de las políticas públicas que más aglutina a la hora de plantear su estructuración y puesta en marcha. Por ende, cuando se trata de programas de política, se destaca que viene trazado por otras entidades o instituciones que no sólo inyectan recursos al programa, sino que lo dirigen y esperan obtener resultados coherentes con su quehacer o estructura de políticas, por cuanto ellas representan en suma, la mejor manera de reconocer la actuación de los Estados.

Tabla 7. Relación programas y organismos para la generación de políticas públicas.

\begin{tabular}{lll}
\hline \multicolumn{1}{c}{ Programas } & \multicolumn{1}{c}{ Organismos } & \multicolumn{1}{c}{ Política Pública } \\
\hline Pobreza & Banco Mundial (BM) & Lucha contra la pobreza \\
\hline Salud & Organización Mundial de la Salud (OMS). & Modulación de Sistemas de Salud \\
\hline Cambio climático & Acuerdo de Cambio Climático )COP - 21) & Mitigación cambio climático \\
\hline Desnutrición & $\begin{array}{l}\text { Organización para la Agricultura y } \\
\text { la Alimentación (FAO) }\end{array}$ & Nutrición y Alimentos \\
\hline Analfabetismo & $\begin{array}{l}\text { Organización de las Naciones Unidas para la } \\
\text { Educación y la Cultura (UNESCO) }\end{array}$ & Cero analfabetismo \\
\hline Educación & Organización Internacional del Trabajo (OIT) & Formación para el trabajo. \\
\hline Económico & Fondo Monetario Internacional (FMI) & $\begin{array}{l}\text { Ajuste económico y fiscal de las } \\
\text { economías o los estados. }\end{array}$ \\
\hline
\end{tabular}

Fuente: Elaboración propia

La posición que logran tomar los proyectos parte precisamente de la coherencia manifiesta entre la planeación, la política y los programas. En este sentido, es propio de los estamentos e instituciones plantear proyectos a escala de ejecución o realizaciones. Dada su periodicidad, estos se asumen como proyectos de política pública, enfocados más a la remediación o intervención de manera puntual en un área de interés. No obstante, es de aclarar que, la trayectoria en el tiempo implica que un proyecto puede pasar a ser subprograma, es decir contenida por periodos, o tomar su propia vigencia con objetivos y recursos como programa, con interacción programática diversa, debido a que existen intereses por temas específicos.

Se ha adoptado tal noción en el entendido de que conviene pensar en las políticas públicas no como un espacio plenamente autónomo, neutro ni sólo de naturaleza administrativa sino más bien concebirlo como una arena donde, si bien converge 
lo multidisciplinario, lo determinante en ella es el factor político: la intervención de actores, proyectos, procesos e instituciones políticas. Martínez (2013:125).

El conjunto de elementos que contiene un proyecto implica toda la trazabilidad operativa que igualmente debe contener la política pública. Inclusive, la versión de proyecto de política pública, es más consistente con la visión de Managment público en la que los impactos y resultados son fundamentales, no sólo para la revisión sino la continuidad de las políticas públicas. Por ende, es de esperar entonces que un proyecto de política pública responda a dichos aspectos, sino que sea contemplado como un contexto técnico de estas. Frente a ello, Debe responderse frente a la estructura el proyecto, objetivos y metodologías, y en consecuencia, con el cronograma, el presupuesto y los referentes.

Tabla 8. Proyectos como política pública

\begin{tabular}{lllllll}
\hline \multicolumn{1}{c}{ Proyecto } & Estructura & Objetivos & Metódica & Cronograma & Presupuesto & Política pública \\
\hline Titulo & Descripción & General & Método & Actividades & Propuesto & Para formular \\
Resumen & Justificación & Específicos & Metodología & Tiempo & Planteado & Para implementar \\
Palabras claves & Preguntas & Hipótesis & Alcances & Ejecución & Aprobado & Para evaluar \\
\hline
\end{tabular}

A diferencia de los planes, políticas y programas, los proyectos de política pública tienen la posibilidad de en la marcha, registrar la posibilidad de organizarlos o evaluarlos e incluso intervenirlos para sacar de ellos los mejores réditos. En este caso, es de considerar que el éxito de los proyectos desde la perspectiva de los mismos, garantizará postular la idea que muchos de ellos terminarían enfilando la hoja de ruta de proyectos con alcance de política pública. No obstante, si perder su naturaleza mediata y neutral, que implica la revisión exhaustiva de los mismos y ello de ser imperante puede llevar a su congelamiento o cancelación definitiva de quienes lo hacen, porque estos se convierten en la vertiente que canaliza el resto de políticas.

En la práctica, las políticas públicas se expresan en los planes, programas y proyectos dirigidos directa o indirectamente por el Estado, donde el contenido de la directriz se despliega de forma detallada y sistemática. Los planes nacionales y sectoriales de desarrollo, los proyectos estatales y los programas de gobierno son mecanismos importantes para la puesta en marcha de políticas públicas y constituyen factores críticos de su éxito. De lo anterior se concluye la importancia de adoptar un modelo apropiado de planificación que soporte la gestión de la política y que defina como mínimo los siguientes elementos nucleares de la directriz: Los objetivos o resultados esperados; las estrategias para lograrlos; los recursos humanos, técnicos y financieros necesarios; los criterios de seguimiento y evaluación. Gómez (2012:24).

La tipología secuencial es la que ha terminado por confluir la mayoría de intereses en materia de formulación, implementación y evaluación de la política pública. Inclusive, puede decirse sin lugar a dudas, que recoge todo lo planteado hasta ahora en cuanto a las otras tipologías de estructuración, disponiendo de los elementos complejos, disipativos y sistémicos para que la política pública salga adelante, tal es así, que al interior de su estructura manifiesta alternativas, supuestos o externalidades que contrarían o jalonan la política pública, escenario explicado por la metodología de marco lógico.

La formulación es un paso vital donde reconociendo ya un problema social se procede a construir la política. Como momento de la secuencia, posee un reconocimiento especial, puesto que es aquí en el que se perfila el modelo de adquisición de decisiones. Este modelo apunta a cómo el decisor elige opciones de políticas para 
la resolución del problema; decisión que tiene lugar gracias a la libertad del actor político que decide o a los factores externos que no puede controlar (Roth, 2002). A continuación, se mencionan los modelos de toma de decisión que pueden tener lugar en la formulación.

\begin{tabular}{|c|c|c|c|c|}
\hline $\begin{array}{l}\text { Modelo de } \\
\text { racionalidad } \\
\text { absoluta }\end{array}$ & $\begin{array}{l}\text { Modelo de la } \\
\text { racionalidad } \\
\text { limitada }\end{array}$ & $\begin{array}{l}\text { Modelo } \\
\text { incremental }\end{array}$ & $\begin{array}{l}\text { Modelo de } \\
\text { la anarquía } \\
\text { organizada }\end{array}$ & $\begin{array}{l}\text { Modelo del } \\
\text { Public choise }\end{array}$ \\
\hline $\begin{array}{c}\text { Autor Frederic } \\
\text { Taylor (1911) }\end{array}$ & $\begin{array}{l}\text { Autor Herbert A. } \\
\text { Simon (1983) }\end{array}$ & $\begin{array}{l}\text { Autor Charles } \\
\text { Lindblom (1959) }\end{array}$ & $\begin{array}{l}\text { Autor March } \\
\text { Olson (1972) }\end{array}$ & $\begin{array}{c}\text { Autores Muller y } \\
\text { Down (1957) }\end{array}$ \\
\hline $\begin{array}{c}\text { Establece } \\
\text { que hay una } \\
\text { solución } \\
\text { superior a otra }\end{array}$ & $\begin{array}{l}\text { Deriva de la } \\
\text { crítica al modelo } \\
\text { anterior }\end{array}$ & $\begin{array}{l}\text { Crítica a los } \\
\text { modelos de }\end{array}$ & $\begin{array}{l}\text { Antítesis de los } \\
\text { modelos de } \\
\text { racionalidad }\end{array}$ & $\begin{array}{l}\text { El decisor } \\
\text { político hace } \\
\text { primar sus } \\
\text { intereses } \\
\end{array}$ \\
\hline $\begin{array}{l}\text { Este modelo } \\
\text { satisface la } \\
\text { razón }\end{array}$ & $\begin{array}{l}\text { El decisor se } \\
\text { encuentra frente } \\
\text { a limitantes } \\
\text { Se adhiere a la } \\
\text { primera solución } \\
\text { que le parece } \\
\text { satisfactoria }\end{array}$ & $\begin{array}{l}\text { La decisión } \\
\text { racional es } \\
\text { inexistente } \\
\text { El decisor } \\
\text { se basa en el } \\
\text { instinto }\end{array}$ & $\begin{array}{l}\text { La decisión } \\
\text { viene marcada } \\
\text { por objetivo } \\
\text { ambiguos e } \\
\text { información } \\
\text { incompleta }\end{array}$ & $\begin{array}{l}\text { Se anteponen } \\
\text { los intereses } \\
\text { políticos por } \\
\text { encima de los } \\
\text { colectivos }\end{array}$ \\
\hline
\end{tabular}

Tomado de: Mejía (2012:148).

El modelo secuencial, permite la combinación de distintas maneras de afianzar, disponer o perfilar la política pública. Goza de la posibilidad de vincular múltiples criterios a la hora de sustentar la política, por eso, tiene gran similitud con el análisis multicriterio, metodología en la que el problema se explica tanto desde lo cuantitativo como de lo cualitativo, y ambos se complementan. Aquí, el tema radica en solventar la política pública con los más diversos argumentos, cifras, datos y elementos para que esta además de explicarse, pueda ser factible, viable y reproducible en los ciclos políticos. Es el que actualmente, tiene mayor ascendencia por la capacidad que tiene dentro de sí, para asimilar otras formas de política.

\section{Fines y funciones del Estado frente a las políticas públicas con enfoque de derechos, legislativas, poblacionales y territoriales}

La política pública tiende a favorecer enfoques mucho más aprehensivos y en línea con los fines y funciones del Estado, en las que cuentan tanto las políticas como la de derechos u otorgamientos a la par con el enfoque legislativo o activismo jurídico, a su vez, que las consecuentes con políticas de enfoque poblacional o integral, como las referentes al enfoque territorial. Dicho conjunto de enfoques, supera la vinculación pretendida de enmarcar a la política pública únicamente desde los enfoques corporativos, gubernamentales o neo corporativos, que son más afines a la concepción de las ciencias políticas.

En este contexto, la alianza entre las organizaciones de desarrollo y aquellas que han adquirido una mayor experiencia en el lenguaje de los derechos y la implementación de estrategia legales, puede aportar miradas complementarias y potenciar la participación social y la fuerza de los reclamos. Abramovih (2004:37).

Es importante afianzar el carácter propio que viene adquiriendo la política pública, cuyo patrón no se instala en un hecho de naturaleza política o de los polices makers. Estas se instalan en el propio orden estatal y en el marco de las divisiones 
de los órganos de poder, como se conoce para los países de naturaleza democrática, como el ejecutivo, judicial y legislativo, quienes de manera concomitante atienden los fines y funciones del Estado. Por ende, la política pública en esencia, no es el acto del político o la política, es el resultado de un proceso mediatico donde conversan diversos actores en su razón de ser con el Estado y lo público, siendo coherentes con el liberalismo.

Por su parte, las políticas públicas, sean cursos de acción o, como menciona Lowi (1972), políticas públicas institucionales, son los mecanismos de acción del Estado desarrollados por el gobierno, a través de procesos por los cuáles la sociedad se organiza. Bajo esta concepción se encuentran integradas instituciones como el Poder Legislativo, el gobierno (Secretarías de Estado) y el Poder Judicial; instituciones que rigen, fijan, modifican o transforman las reglas o normas que tutelan el estatus de personas físicas y morales, y el régimen jurídico de sus bienes y derechos. Valencia (2012:13)

La política pública con enfoque de derechos está orientada a evaluar la relación contractual entre el Estado y los súbditos, en cuanto al marco creado por el orden constitucional desde el cual se deriva el Estado Social de Derecho. En dicho escenario, se insta para que a medida que el Estado evoluciona los derechos sean evaluados o replanteados en tanto la capacidad y naturaleza misma del momento que registra este. No se trata de una extensión o entronización del deber ser del Estado, se trata de mediar la relación con los ciudadanos por la vía de la provisión de políticas públicas, a manera de recambios en su accionar.

Si, además, agregamos que estas políticas públicas deben tener un enfoque de derechos, necesitarán, incluso más que cualquier otra, contar con la participación de la ciudadanía en todo su ciclo; entregar garantías a la población respecto a los derechos que están considerados en ella; y haber sido lo suficientemente informadas y difundidas para que las personas a las cuales están dirigidas puedan hacer ejercicio de dichos derechos. Ochoa (2011:7).

Es evidente que la política pública con enfoque de derechos implica el reconocer el contrato social del Estado con los ciudadanos. En esto, no basta la carta constitucional, ni el refrendar o solicitar mejoras a la carta. En ellas, el preámbulo, capítulos o parágrafos no es una recreación del posible Estado a construir, es un contrato para cumplir. En el caso colombiano por ejemplo, no es claro el contrato social del Estado para con los ciudadanos, dado que no se han resuelto referentes a los fines del Estado, en tanto el poder del derecho, la aplicación de la legislación, el reconocimiento a la población y el territorio.

Pese a que la referencia al Derecho en la teoría de las políticas públicas es escasa, en Colombia ha crecido el interés jurídico en este tema en los últimos años. En la vía inversa, los analistas de políticas públicas han prestado mayor atención al Derecho -y a los derechos- más allá de asumirlo como el marco jurídico institucional de toda política. Una de las razones de esta reciente conexión es la reiterativa referencia al concepto de políticas públicas en la jurisprudencia constitucional colombiana. Cano (2014:435).

La política pública con enfoque legislativo o activismo jurídico goza de plena valía en la medida en que no se concreta el contrato social y emerge el extracontractualismo en el que los diversos actores, incluyendo el Estado, no solo buscan otorgar o reivindicar sino encuadrar los fines y funciones del Estado. En este ámbito, las Cortes, sean estas constitucionales, de estado o jurisprudencia al igual que el legislativo, se 
dedican no solo a enmendar o dar respuesta a las disquisiciones entre los actores, sino de disponer inmediatamente de la acción a seguir. Es decir, que el legislativo, inclusive la rama judicial, más que el político, es un hacedor de políticas públicas.

\section{Los escenarios fácticos de las políticas públicas en la jurisprudencia}

Se identificaron dos escenarios fundamentales en los que la Corte Constitucional alude a las políticas públicas: i). Cuando reconoce la necesidad de formular políticas que desarrollen la faceta prestacional de los derechos y ii). Cuando declara un estado de cosas inconstitucionales (ECI). Ambos escenarios no son excluyentes, es decir que es posible encontrar ambos argumentos en una misma providencia, sin embargo estos contextos se separan en el escrito para resaltar que no siempre que la Corte adopta órdenes complejas y fallos estructurales -que involucran políticas públicas- se ha declarado un ECl. Cano (2014, p. 440-441).

El denominado activismo jurídico, es un claro promotor de políticas públicas en las que se instala el marco extracontractual en la que el Estado como los ciudadanos, busca equilibrios o ajustar situaciones que no pudieron resolver por la vía del enfoque de derechos. Mientras que este último se enmarcar por la vía del justo medio, en el que se busca el beneficio para la mayoría, el enfoque legislativo fomenta el justo precio, en el que tanto la ley como la economía juegan a la hora de conciliar las discrepancias, en tanto, lo solicitado como los recursos, se convierten en unidad de materia para el hacedor legislativo.

En este sentido, las decisiones legislativas, al tiempo que concilian intereses sociales y políticos de corte coyuntural, son decisiones colectivas cuya base se define en términos de objetivos nacionales y/o permanentes plasmados en la Constitución. Puede decirse que la decisión legislativa significa la adecuación motivada y fundamentada de las instituciones con base en consensos que establecen lo que se considera deseable, factible y socialmente aceptable. Las modificaciones normativas (a la constitución, a las leyes, a los tratados, a los reglamentos) producen cambios en los valores y en los procedimientos organizativos. Valencia (2012, p.13).

La interacción entre el enfoque de políticas públicas de derechos y el enfoque legislativo, se advierte en la medida en que el primero se ubica en el contexto de las demandas (justo medio), y el enfoque legislativo en el de la oferta (justo precio). De manera clara, puede decirse que la interacción entre ambos frentes hace posible conciliar el orden contractual y extracontractual del Estado en la medida que las políticas públicas que emergen en ellos superan cualquier discrecionalidad política e incluso pública, dejando al Estado y a los órganos de poder en el centro, y esta es la tendencia que se está generando.

En esta primera situación, el camino adecuado que a juicio de la Corte debería seguir una ley, es ser desarrollada por el ejecutivo mediante una política pública a partir de los lineamientos legislativos. La política, por tanto, hace parte de la implementación de la ley. En la segunda situación hallada en otro grupo de decisiones, la Corte estima que en ciertos temas existe una especie de reserva de ley para formular la política pública. Este escenario aparece respecto a las políticas de seguridad y defensa y frente a la política criminal. Cano (2014:451).

El enfoque de políticas públicas con enfoque poblacional trata de perfilar las funciones del Estado más que los fines. En este ámbito, se encuentran la asignación de recursos, la distribución de la riqueza, la estabilización económica y política y el 
fomento al desarrollo, dejando en la actualidad apertura para los primeros y reserva para los segundos. En este enfoque, la población se convierte en hacedor de políticas públicas e incluso orientador de esfuerzos y recursos del Estado, para bien de los intereses de las poblaciones, en la que se considera que la co-gestión hace más eficiente la política pública.

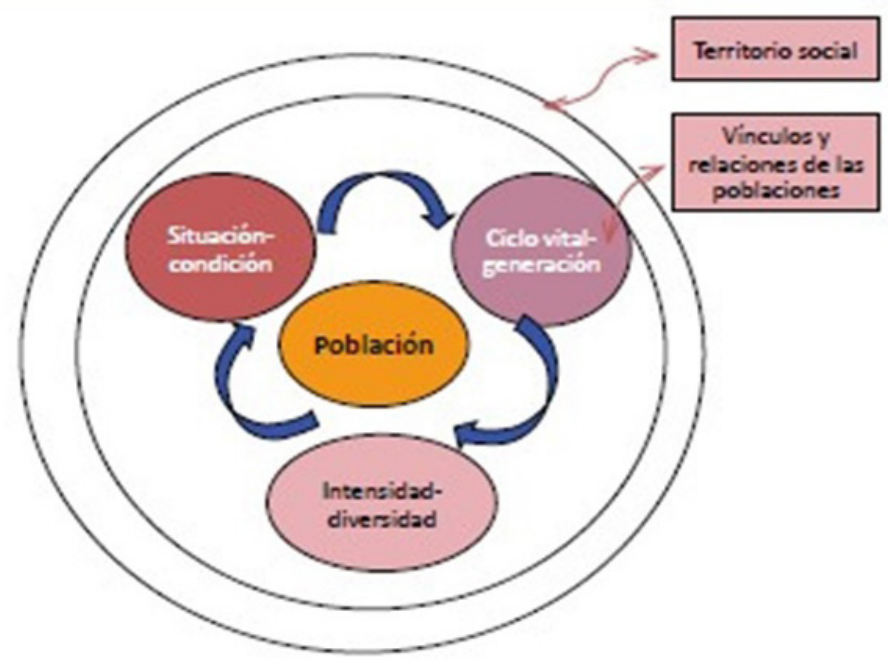

Esquema 2. Propuesta de enfoque poblacional. En: (García, 2010, p.11).

La población pasa a ser hacedora, ejecutora y quien plantea las políticas públicas, en una especie de Estado de mayor evolución o liderazgo, desde el cual se generan las condiciones para que la población líderes procesos de política pública por encima de la cosificación a la cual la política quiere darle a la política pública. En este ámbito, se requiere de una reconformación de las instancias de participación, que favorezcan no solo la presencia de la población, sino que su participación sea verificada con la puesta en marcha de proyectos o programas de políticas públicas ejecutadas por la población.

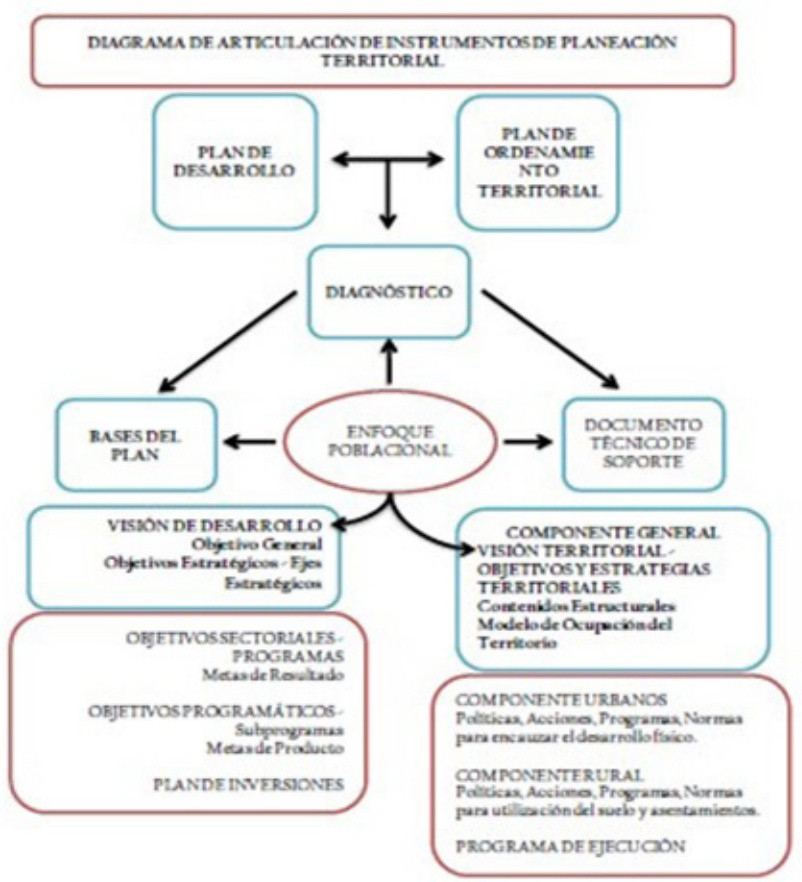


Así mismo, desde el punto de vista estructural, una vez integrado el enfoque poblacional, a los aspectos estratégicos propios de los instrumentos de planeación territorial, estos serán transferidos en cascada, permeando los objetivos y metas, tanto de resultado como de producto y por consiguiente, su relación con el marco de inversiones para el desarrollo. En: Hurtado y Agudelo (2012:11).

La política pública preeminente gira en torno a la interacción entre el Estado, la población y los agentes interesados en desarrollar o llevar a cabo temáticas de interés general o particular. Es decir que, la política pública ha dejado de encumbrarse en la política para convertirse en un escenario de uso generalizado por quienes consideran que es un instrumento valioso, capaz de hacer converger las disparidades que el enfoque de derechos y el legislativo no alcanzan a contemplar, por cuanto, termina por ubicar sus propósitos en la población, cumpliendo la regla de la mayoría.

En este sentido, el tipo de análisis aquí propuesto concibe a las políticas públicas no sólo como un curso de acción deliberadamente diseñado, también como el curso de acción efectivamente seguido; no sólo lo que el gobierno dice y quiere hacer, también lo que realmente hace y logra, por sí mismo, o por interacción con actores sociales y políticos, más allá de las intenciones declaradas en normas, leyes, planes y programas. Habla entonces, no del deber ser, sino de políticas públicas realmente existentes. 0 , en otros términos, primero lo descriptivo-analítico y luego lo prescriptivo. Naranjo et al (2009:83).

El desenvolvimiento de las políticas públicas en su versión funcional centradas en sus finalidades, impactos y resultados son los elementos que favorecen la adecuación de las "nuevos enfoques de política pública, centrados en el Estado y sus órganos de poder". Bajo dicho cometido, el territorio emerge como la plataforma de ocupación de la población, pero en el que igualmente se asientan las políticas públicas, por ende, el enfoque territorial tiende a focalizar no solo los mayores y mejores recursos sino en donde se pretende que tenga sentido la política pública a materializar, dando margen para su eficacia y eficiencia.

La gestión territorial de la política pública entrama entonces una concatenación económica, político-administrativa, sociocultural, institucional y técnico-productiva, en la concertación de actores. La práctica se sustenta en la cooperación y fomento de capacidades para la autogestión, en el entendido de que organización y diligencia pública trascienden la estructuración de las políticas, para concretar la institucionalidad de lo territorial. (González, 2013, p.246).

Es decir que la política pública pasa de ser direccional a hacer consultiva de los territorios en la medida en que se ubican en el espacio - lugar para enfocar el tránsito de estas en ellos. Se trata de una manera un tanto audaz de promover la idea de que las políticas públicas no pueden ser replicas y que por tanto tienen asidero propio, provocado entre otras por el territorio. En este sentido, más que el plan, los programas o la política, es el territorio quien termina activando o priorizando las políticas públicas como eje articulador con el Estado, las instituciones o los diferentes agentes, sobre todo en lugares en donde se requiere recuperar primero el territorio antes que lo institucional. 


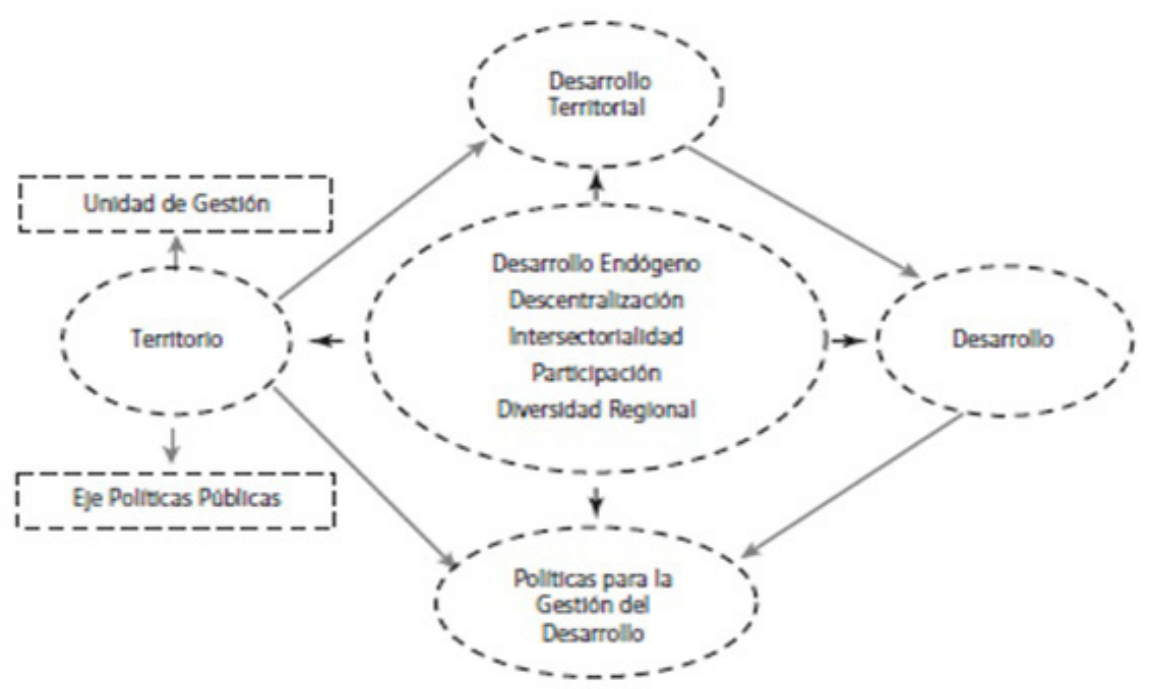

Esquema 4. Modelo de gestión para el desarrolloterritorial. En: (González, 2013, p. 248).

Finalmente, es factible decir que en los lugares como departamentos o municipios con dificultades gubernamentales o institucionales o de administración pública, lo primero que debe rescatarse es el territorio como referente de política pública, por ende, los planes, políticas, programas e incluso proyectos, no tienen mayor norte cuando el territorio no se reconoce como instancia para favorecer la co-creación de diversas temáticas. En este sentido, la política pública con enfoque territorial lo que infiere, es que su centralidad es más favorable en entidades administrativas en las que se tienen dificultades como las planteadas al inicio, dado que es allí donde se podrán evidenciar apliaciones conforme a la praxis de las mismas en cuanto a aplicabilidad no solo por orden de importancia sino por desarrollo y desencadenamiento consecutive de acciones de éstas.

\section{Conclusiones}

La política pública, por su propia naturaleza ha terminado por regresar a su espacio natural, como es el Estado y los órganos de poder, donde se encuentra una interrelación entre política - politico - público. Por tanto, el desviar la atención al origen político o de las políticas, es solo cosificar el papel que tienen estas para que los Estados puedan cumplir sus fines y funciones a cabalidad, en razón de que, recae en los derechos, la legislación, la población y el territorio incubar la posibilidad de que las políticas públicas tengan asiento realmente en cuanto a su función especial de ser canalizadoras en determinada forma de los actores - ideas - proyectos, que realizan al fin de cuentas, acciones desplegadas de contribución al cumplimiento d eobjetivos transversales entre lo Estatal (macro) y territorio (micro), siendo este ultimo un detonante clave de éstas según el grado de uso e importancia que se les de. Dado que de lo contrario, erroneamente se pueden convertir inconcientemente estas en un memorial de agravios, peticiones o reclamaciones, lo cual las aleja de su esencia funcional.

El ciclo de políticas públicas, corresponde precisamente a la revisión sobre el estado de fenecimiento o eliminación de las políticas públicas que puede ser por su propia naturaleza o por consecuencia lógica de su inoperancia. Por ende, no todo puede tener el remoquete de política pública o no todo tema tiene la capacidad para cargarse tal manifiesto. Por ende, antes de priorizar cualquiera de ellas, debe considerarse qué tanto asentamiento o campo de acción tienen estas en la causalidad racional de 
donde se planearán y desarrollarán. Además, es válido expresar que al reconocerlas como instrumentos, se elimina el carácter de orden general que se le quiere dar estas por encima del Estado o los órganos de poder.

En cuanto a los enfoques tradicionales, enfocados en los planes, políticas, programas o proyectos, estos son útiles en la medida en que se resuelve el otro lado de las políticas en tanto los fines y funciones. Son trascendentales, en la medida en que cada política pública consulte el marco contractual y capacidad extracontractual que tiene todo el aparato estatal frente a los agentes, por ello, es que el matiz económico ha tomado gran fuerza a la hora de exponer lo que realmente implica la consumación o puesta en marcha de una política pública, tanto, que ha terminado por enfilar cualquier orientación que se quiera hacer.

No obstante, sin importar la orientación que se de dictada sea por el entorno mismo (matices) o por los agentes en sus períodos de realición y consecución de objetivos de agenda, se debe reconcoer que la política pública es un aspecto que debe ser comprendido primeramente desde sus consideraciones etimológicas en conceptos, para luego llevarlas eficazmente a la p'raxis continua de las mismas, donde esta misma acción determinará finalmente que el objeto final de su uso sea una simple actividad recurrente por agenda o motivos secundarios que se dieron (como ha sucedido en ocasiones), como podrá ser finalmente un objeto causal acorde a una planeación que desencadene un desarrollo, seguimiento y demás factores consecuentes que permiten que esta transcienda a largo tiempo, lo cual traerá utilidades de mayor alcance que las reflejadas al corto plazo (si son solo actividades recurrentes) que demuestran es resultados de costo/beneficio.

Sin embargo, considerando la segunda apreciación sobre el transcender de la misma prolítica pública en un orden lógico de que primeramente debe ser comprendida en concepto y luego en práctica del mismo, cabe anexar que el objeto final de la práxis solo será verídico en efectividad mediante la indexación causal del territorio, donde se comprende el entorno en materia de prioridades, es decir, qué require la comunidad y cómo el Estado o su entidad de administración territorial representativa puede convegir en relación a los habitantes su desarrollo de políticas públicas precisas, cumpliendo así sus compromisos sociales y de gestión de recursos.

\section{Referencias}

Abramovih, V. (2004). Una aproximación al enfoque de derechos en las estrategias y políticas de desarrollo de América Latina. Centro de Estudios Legales y Sociales, Argentina). Documento preparado para: Derechos y desarrollo en América Latina: Una reunión de trabajo. Santiago, Chile, Diciembre 9 y 10 de 2004. 1-39.

Cano, L. F. (2014). La narrativa de las políticas en la jurisprudencia de la

Corte Constitucional colombiana. Pap. Poli. 19 (29; 435- 458.

Cuervo, J. I. (2007). La definición del problema y la elaboración de la agenda.

Ensayos sobre políticas públicas. Universidad externado de Colombia. $1^{\text {a }}$ edición. 151-165.

Echeverri, R., González, H., Echeverri, A., y Miranda, A. (2011). La Institucionalidad de lo territorial gestión y política pública. En: J. Forero, G. García Ruiz, Solángel 
et, al (2010). El enfoque poblacional: las personas como centro de las políticas. Revista de investigaciones en Seguridad Social y Salud. (12), 129-142.

Gordillo y A. González (eds.), Mundos rurales y transformaciones globales: desafíos y estrategias de respuesta (pp. 1-24). Bogotá D. C.: Pontificia Universidad Javeriana.

Gómez R (2012). Gestión de políticas públicas: aspectos operativos. Rev. Fac. Nac. Salud Pública, Vol. 30 No.. 2, p. 223-236.

González, J. et al. (2013). La territorialización de la política pública en el proceso de gestión territorial como praxis para el desarrollo. Cuadernos de Desarrollo Rural, Vol. 10, No.72, 243-265.

Gris, P. C. (2016). Trascendiendo la instrumentalidad: metodología y evaluación. Vol. 7, p. 1-27.

Hurtado, S. P. y Agudelo, S. (2012). El enfoque poblacional, una nueva visión de la planeación y el desarrollo territorial. Lineamientos para la formulación del plan de desarrollo. Gobernación de Antioquia. Secretaría de Planeación del Quindío. 1-25.

Jolly, J. F. \& Cuervo, J. I. (2007). Introducción. La posibilidad de las políticas pùblicas.15-24. Economía y políticas públicas. Ensayos sobre políticas públicas. Universidad externado de Colombia. $1^{\text {a }}$ edición.

Jolly, J. F. (2007). Economía y políticas públicas. Ensayos sobre políticas públicas. Universidad externado de Colombia. $1^{\text {a }}$ edición. 131-145.

Lahera,. E. (2004). Política y políticas públicas. Serie División de Desarrollo Social. Políticas Sociales (95), 1-33.

Lowi, T. J. (1972). "Four Systems of policy, politics and choice", en Public Administration. Review 32 (4), 298-310.

Martínez, M. I. (2013). Una propuesta de modelo para la evaluación de los proyectos de cooperación al desarrollo basado en el análisis de políticas públicas. Universidad Autónoma de la Ciudad de México. Andamios. Revista de Investigación Social. 10 (21), 119-150.

Mejía, J. (2012). Modelos de implementación de las políticas públicas en Colombia y su impacto en el bienestar social. Universidad Pontificia Bolivariana. Analecta. 2 (3), $141-164$

Naranjo, G.; López, J. E. y Granada, J. (2009). Las políticas públicas territoriales como redes de política pública y gobernanza local: la experiencia de diseño y formulación de las políticas públicas sobre desplazamiento forzado en el departamento de Antioquia y la ciudad de Medellín. Estudios políticos. (35), 81-105.

Ochoa, G. (2011). Políticas públicas con enfoque de derechos y organizaciones no gubernamentales en Chile. Germina, p. 1-28.

Ochoa, G. (2008). El sector público y la participación ciudadana. Recuperado el 10 de marzo de 2011 desde www.germina.cl. 
Roth, A. N. (2007). Enfoques y teorías para el análisis de las políticas públicas, cambios de acción pública y transformaciones del Estad, p. 29-63.

Roth, A. N. (2004). "Políticas Públicas. Formulación, Implementación y Evaluación". Ediciones Aurora, Bogotá, D. C.; agosto de 2004. Páginas 29 a 49: Enfoques Teóricos y Tipología de las Políticas Públicas.

Roth D, A. N. (2002). Políticas públicas: formulación, implementación y evaluación. Bogotá. Editorial aurora.

Sour, L. (2008). El enfoque económico en el estudio de las políticas públicas. Universidad de los Andes. CIDE. 1-24.

Valencia, L. (2012). La relación ejecutivo - legislativo en la elaboración de políticas públicas. Instituto Tecnológico y de Estudios Superiores de Monterrey. Confines de Relaciones Internacionales y Ciencia Política. Vol. 8, No. 16, p. 11-37. 